\title{
Comentarios y Lenguas Vernáculas: La Traducción como vehículo cultural y propagandístico
}

\author{
ROXANa RECIO \\ Creighton University
}

Normalmente cuando se habla de los comentarios medievales, no se trata de las traducciones de las mismas. Por otra parte, la mayoría de los estudios que existen se aproximan a los comentarios desde un ángulo formal: las partes, la ideologia, la manera en que el comentarista la ha escrito. Se trata de un punto de vista retórico. Además se han estudiado mucho más los comentarios en latín que los que están escritos en lenguas vernáculas.'

La critica ya ha demostrado la relevancia de las traducciones en la literatura didáctica y en algunos textos con comentario. A lo largo del siglo XV el texto glosado, especialmente el que traducía de una obra originalmente en latín, era algo muy común. Esto se aprecia en traductores como Pero López de Ayala, Alonso de Cartagena, Enrique de Villena, Fray Alonso de Cristóbal y Gómez García del Castillo, entre otros ${ }^{2}$. También puede verse el mismo asunto en las traducciones que se hicieron de Illicino, de Vellutello y de Landino, tanto en castellano como en catalán. En este trabajo voy a analizar los traductores y las traducciones de esos comentaristas que se ocuparon tanto de Petrarca como de Dante.

A este efecto llevaré a cabo un cotejo entre los textos y utilizo para ello manuscritos y ediciones que provienen de diferentes bibliotecas: la Biblioteca Nacional de Madrid, la Biblioteca Nacional de París, la Biblioteca de Catalunya en Barcelona y la Biblioteca de Cornell University. No se trata de un cotejo a nivel linguístico, como tradicionalmente se ha venido haciendo con las traducciones, sino que voy a investigar la dimensión de su carácter cultural, lo que lleva a ocuparse de cuestiones relacionadas con la sociedad y el tipo de lector al que van dirigidas las obras.

1 Prancisco Rico, Alfonso el Sabio y la General Estoria, Barcelona: Ariel, 1984, 167.

2 Julian Weiss, The Poet's Art: Lizerary Theory in Castile c. 1400-1600, Medium Aevum New Series 14, Oxford: Oxfond University Press, 1990, 121. 
Por otra parte, hay que especificar que el concepto de comentario lleva irremediablemente a hablar de «amplificación» puesto que es un utensilio indispensable para muchos comentaristas. No obstante, como me he ocupado de «la amplificación» en otro trabajo, aquí trataré ese concepto de una manera muy limitada ${ }^{3}$.

El comentario no respondía solamente a una labor didáctica o explicatoria. Algunas veces con un comentario se embellecía un texto, realzando imágenes y ofreciendo explicaciones psicológicas ${ }^{4}$. En lo que al campo de la traducción se refiere, el comentario está relacionado con dos factores fundamentales: el desprecio hacia la lengua vernácula y la aceptación de traducciones oscuras.

Hay que tener en cuenta que las lenguas romances, por venir del latín, eran inmediatamente consideradas inferiores. Al considerarse el latín una lengua perfecta y, más aún, al considerársele el origen de todas las lenguas romances, se tendía a ver como imperfectas y faltas de capacidad lingǘstica para expresar los conceptos latinos a todas las lenguas romances. Por eso, cualquiera de estas lenguas romances, incluyendo el castellano, cada vez que servían de vehículo para expresar conceptos latinos necesitaban de grandes explicaciones, puesto que ellas no tenían conceptos equivalentes a los latinos. Peter Russell ya ha dado bastantes testimonios como ejemplo ${ }^{3}$. Entre los ejemplos de Russell, además de Cartagena, destacan latinistas como Alfonso de Palencia. Sin embargo, esta actitud en relación a la lengua vernácula y el latín no la encontramos sólo entre latinistas y teóricos, sino que también la compartían los poetas más famosos de la Castilla de aquel tiempo. Por ejemplo, Juan de Mena, en su prólogo a la traducción de la Yliada ${ }^{6}$, sigue a Cartagena en aceptar la pérdida en romance de la «dulzura» latina, pues este ya lo había especificado en su Rethorica?

Esta actitud cerrada chocaba con las ideas de San Jerónimo sobre la traducción $^{8}$. Hace ya algunos años Margherita Morreale hablaba de una corriente liberal patrocinada por el santo ${ }^{\circ}$. Esta corriente es algo indiscutible a la luz de la tradición sobre la traducción. Dice San Jerónimo: «Sensum exprimere de sensu» ${ }^{10}$. El interés estaba cifrado en el sentido del texto, no en las palabras. Las ideas eran lo fundamental. El verdadero traductor según San Jerónimo es aquel que es capaz de entender en su propia lengua el significado del texto del que se traduce.

${ }^{3}$ Roxana Recio, «Approaches to Medieval Translation in the Iberian Peninsula: Glosses and Amplifications», Fifteenth-Century Studies 1998, en prensa.

4 Rico, $183-87$.

s Peter Russell, Traducciones y traductores en la Peninsula Iberica (1400-1550), Bellaterra: Universitat Autónoma de Barcelona, 1985. Especialmente los cuatro primeros capitulos, 5-26.

6 Juan de Mena, Obras completas, ed. Miguel Angel Pérez Priego, Barcelona: Planeta, 1989, 334.

7 Alonso de Cartagena, La Rethorica de Marco Tullio Cicerón, ed. Rosalba Mascagna, Napoli: Liguori, 1969, 31.

- Para ver más testimonios con respecto a esta postura de los latinistas frente al castellano puede consultarse a Julio César Santoyo, Teorla y crítica de la traducción: Antología, Bellaterra: Universitat Autonoma de Barcelona, 1987, 23-44.

9 Margherita Morreale, Castiglione y Boscdn: el ideal cortesano en el renacimiento espantol, 2 vols., Madrid: BRAE, 1959, vol. 1, 19.

10 Saint Jérome, «A Pammachius; la meilleure méthode de traduction», Lettres, ed. Jerome Labourt, 8 vols., Paris: Les Belles Lettres, 1953, vol. 3, 59. 
Esto nos lleva a tratar el segundo de los factores arriba mencionados: las traducciones oscuras. A medida que se va adentrando el siglo XV, se siente la necesidad de romper con la oscuridad de los textos y se intenta hacer más claras y familiares las traducciones. Poco a poco penetran los autores y las ideas italianas, humanistas. Dentro de este proceso cobran sentido retrospectivamente algunas manifestaciones de Enrique de Villena". De alguna manera habla que suplir esa falta de «dulzura y gracioçidad», o sea, de la elocuencia latina en las traducciones, dado que en éstas, como decía Alfonso de Palencia, «lo agudo se torna grosero y lo muy vivo se amortece del todo ${ }^{12}$. Es precisamente en el intento de seguir a San Jerónimo sin romper con la autoridad de los latinistas cuando se utiliza el comentario en función de las traducciones. Cada vez hay una necesidad más imperiosa de que la traducción quede clara, es decir, entendible y familiar, cercana al lector peninsular. Ahora es cuando en muchas ocasiones la amplificación será un factor decisivo con respecto a la naturaleza de la traducción.

La utilización del comentario está en función del tipo de traducción que se lleva a cabo. Se entra en otra dimensión, la de la problemática de las traducciones que se ajustan o se distancian del texto base, $o$ en otras palabras, la problemática de las traducciones literales o libres ${ }^{13}$.

Con respecto a estos dos tipos de traducciones el testimonio más conocido que nos ha llegado es el de Alfonso de Madrigal. Ya es sabido que El Tostado distingue entre la interpretación, es decir, traducción ajustada al texto base, y la glosa, la traducción que permite cambios, añadidos y supresiones, la propia de «menores ingenios». Como creo haber demostrado en otra parte, esta distinción es artificial y responde a un acto de respeto en relación a las teorías establecidas con respecto a la fidelidad al texto por parte de los latinistas ${ }^{14}$. La obra de la que se traducía era ya considerada otro mundo aparte. Por ese motivo se aceptan todos los cambios necesarios en el transvase, y en esos cambios se incluyen las amplificaciones y las explicaciones pertinentes, pero ya como parte del andamiaje de la traducción en sf. Esto le daba libertad al traductor al presentar su texto ${ }^{15}$.

En el ámbito peninsular aparecen dos tendencias a lo largo del siglo XV en el campo de la traducción. La primera se desarrolla más en la Corona de Aragón. Existen traducciones ajustadas al texto base y otras de carácter independiente en donde predomina el criterio del traductor. Así nos encontramos con traducciones como las de la Fiammetta de Boccaccio ${ }^{16}$ o la Cárcel de Amor de Diego de San

"Me ocupo de Villena en un trabajo aparte: «'Por la orden que mejor suena': traducción y Enrique de Villenax, La Corónica 24.2, Spring 1996, 140-53.

12 Alfonso de Palencia, Batalla campal de los perros, Sevilla: 1490, fol. aij(r). Cito de Peter Russell, 29.

${ }^{13}$ Russell da un panorama general del asunto. Véase especificamente el capitulo 5, 26-35.

14 «Alfonso de Madrigal (El Tostado): la traducción como teoría entre lo medieval y lo renacentista*, La Corónica 19.2, 1992, 112-131.

is Para estos aspectos de El Tostado véase también mi artículo «El concepto de la belleza de Alfonso de Madrigal (Bl Tostado): la problemática de la traducción literal y libre», La traducción en España (ss. XIV-XVI), ed. Roxana Recio, Leon: Universidad de Loon, 1995, 59-68.

16 Estoy terminando un trabajo sobre esta traducción. 
Pedro $^{17}$. Esto en las traducciones de vulgar a vulgar viene facilitado por la cercanía lingulfstica. No obstante, hay otras traducciones muy libres, de las que tambien ya he hablado en otros lugares, como la anónima catalana del Decame$r^{\prime} n^{18}$. Por esa independencia del traductor esta traducción ha sido considerada por la crítica una traducción mala. Otros casos curiosos son los comentarios y las paráfrasis de Dante, como la de Ferrer de Blanes ${ }^{19}$.

Quizá tanto los casos curiosos como las producciones de traducciones ajustadas al texto base en Aragón son posibles gracias a la existencia de una larga tradición que comenz 6 con los predicadores, afect 6 a historiadores como Antoni Canals y luego pas6 a la literatura como, por ejemplo, la Vita Christi de Isabel de Villena ${ }^{20}$. Si el caso de Canals es interesante, el caso de Villena no deja de ser menos. Las explicaciones que de la vida de Cristo da Sor Isabel no solamente atañen a cuestiones de traducción, amplificación o glosa y a la utilización del latín en el marco de una producción en romance, sino también a la utilización de un lenguaje familiar en función de un público determinado.

La segunda tendencia peninsular aparece sobre todo en Castilla en donde, a pesar de lo ya escrito por El Tostado, prevalece la autoridad de los latinistas y no se acepta del todo a San Jerónimo. Aquí abunda el comentario. La raíz es toda una gama de preceptos de cómo traducir el verso y la prosa.

Pero las ideas sobre traducción van cambiando a medida que transcurre el siglo. Cada vez son más los traductores que aceptan a San Jerónimo en Castilla, incluso traductores de obras escritas en latín. Un ejemplo de esto lo ofrece Peter Russell cuando nos deja saber que el príncipe de Viana recurre al santo para la defensa de su traducción de la Ética de Aristóteles, la cual tenía como base la versión en latín de Bruni ${ }^{21}$.

En los traductores de los comentaristas italianos esta problemática está presente. Por ejemplo en el caso de Illicino las aproximaciones de los traductores peninsulares son muy particulares. Un ejemplo lo tenemos en la traducción catalana de I Trionfi del siglo XV. Aqui, el comentario de Illicino, cuando introduce la explicación de los seis triunfos, se presenta de una manera escueta y con un vocabulario muy moral. Precisamente esa presentación de los triunfos, de la que ya he hablado en otro lugar ${ }^{22}$, es la que da paso a su conclusión. Si miramos con

17 Para esta obra véase mi trabajo \&La literalidad y el caso de la Carcel de Amor: el quehacer del traductor catalán y del traductor italiano», Hispanic Journal 17.2, Fall 1996, 271-83.

is «Del latín al vernáculo: la difusión peninsular del Decameronw, Livius 9, 1997, 109-19.

19 Me he ocupado de este autor y de su obra en dos trabajos: «Humanismo y exegesis medieval: el caso de Ferrer de Blanes*, Actas del XII Congreso de la Asociación Internacional de Hispanistas; Birmingham, 1995, ed. Aengus M. Ward, 5 vols., Birmingham: The University of Birmingham, 1998, vol. 1, 293-301; y «La interrelación intelectual en la Península: Santillana y Ferrer de Blanes», Anuario Medieval 6, 1994, 159-73.

20 Trato de estas cuestiones en «Las interpolaciones latinas de Sor Isabel de Villena: ¿traducciones, glosas o amplificaciones?», Anuario Medieval 5, 1993, 126-40.

21 Russell, 29.

2 Roxana Recio, «Puntualizaciones sobre la traducción catalana del Triunfo de Amor de Petrarca según el manuscrito 534 de la Biblioteca Nacional de París», Actas del XIII Congreso de la Asociación Internacional de Hispanistas; Madrid 1998, en prensa. 
detenimiento el texto italiano se ve el intento de Illicino de ofrecer una explicación de la obra de Petrarca más filosófica que poética o literaria en general, con la intención de poner en primer plano una connotación cristiana ortodoxa:

Explanato adunque qual sia l' universale soggetto del libro conueniente cosa e condescendere hormai a vedere qual sia la vtilita che ne contribuisce la presente dottrina. Se vera e la sententia de tutti gli moraliche le operatione virtuose e vera essa virtu sia sommo bene e quello si diffinisca essere utile che per dextra via si conduce a la possesione di tal bene. Certamente nessuna cosa ad noi po dimostrarsi piu vtile che la presente dottrina. Imperoche chi fara quello che conosciuta la ignobilita de le illecebre humane inteso etiamdio la excellentia del ragione uolmente operare non sottometta lo sfrenato appetito a la moderata ragione $e^{23}$.

La traducción catalana del pasaje es la siguiente:

Explanant demonstra donchs que tal sia lo universal subieste del libre conuenient cosa es condecendre a veure qual es la utilitat que en la present doctrina contribuyex. Si bera es la doctrina de tots los morals que les birtuoses operaçions e aquesta birtut sia sobixan be allo se determene esser util que por recta bia a la possessio de tal bens porre certament neguna cosa da nosaltres mes util que la present doctrina por demostrarse. Empero quy sera aquell que coneguna la ignobilitat de la humana operaçio entesa encara la excellencia del rahonahement obrar no so tiniesta lo desfrenat apetit a la moderada raho ${ }^{24}$.

Como puede observarse, no existen alteraciones, intervenciones del traductor, amplificaciones, $o$ añadidos de cualquier indole. Tampoco existen explicaciones, ni tan siquiera sobre algunas de las palabras. Estamos ante lo que se suele llamar tradicionalmente una traducción literal de Illicino, dado que las palabras son casi las mismas y existe la misma connotación dialéctica. Resulta ahora interesante ver cómo aparece este pasaje en la traduccion castellana de 1512. En esa fecha Antonio de Obregón también traduce por primera vez los seis triunfos de Petrarca al castellano $0^{25}$. Dentro de la sección que acabamos de ver tanto en italiano como en catalán, hay cambios sustanciosos que afectan al traslado como tal y a la ideología. Obregón aclara, lo que convierte su traducción del comentario en algo singular, dado que hay ya una intervención personal de su parte. Entonces, no puede hablar de todas las cosas que hablan los antiguos y resumirá todo el asunto en cuatro conceptos: 1) «sugeto» y materia de la obra; 2) utilidad de la misma; 3) explicación del origen del autor a través de su nombre, y generalidades del texto que presenta al lector castellano; 4) división de los capítulos. Lo que hay que destacar aquí con respecto a cómo se traducían los comentarios en la Península Ibérica es que, a diferencia del traductor catalán, Obregón

23 Bernardo Illicino, Triomphi de meser Francesco Petranca, Venezia: 1475, fol. a2. Todas las citas seran de esta edición.

24 Francesco Petrarca, Triumphi, ms. 534 de la Biblioteca Nacional de Parfs, fol. ii.

${ }^{25}$ Antonio de Obregón, Francisco Petrarca, con los seys triunfos del toscano sacados en castellano con el comento que sobrellos se hizo, Logrotio: Amau Guillén de Brocar, 1512. Las citas serán de esta edición. 
reelabora todo el pasaje de Illicino introduciendo en la explicación de los distintos triunfos la filosofía, la moral y la doctrina ortodoxa. Si en el texto italiano, y en el catalán, son dos partes diferenciadas, el comentario en castellano lo presenta todo junto. Resulta inevitable pensar en un público diferente. El texto castellano es el siguiente:

Quanto a la intencion y sugeto que es la primera digo que considerando nuestro poeta seys estados de nuestra vida compuso seys triunphos con muy artificiosa fiction poetica. El primero de los cuales es el de Amor, porque naturalmente en la juuentud son todas las gentes sugetas al apetito sensitiuo y, porque en la semejante edad tiene la sensualidad su sefiorio muy poderoso, finge nuestro poeta que venia amor sobre un carro triunphal con infinitos presioneros en muy estrechas presiones. El segundo triunpho es de la Razon o castidad, donde en persona de la razon viene figurada madona Laura trivnphando del amor despues de haverle vencido, lo qual naturalmente acaesce en la edad perfecta y en la vejez quando los deleytes sensitiuos se an resfriado por auer el calor natural perdido parte de sus fuerças, y avn porque los hombres vienen a caer en el verdadero conoscimiento y emiendan los yerros passados con las virtudes presentes y desta manera trivnpha la razon de la sensualidad. El tercero triunpho se llama de muerte, porque, como los hombres llegan a la virilidad y despues a la vejez obrando virtuosamente y teniendo sometido la voluntad a la razon, llega la muerte y acaba las vidas de los mortales quedando ella en el campo como vencedora vniuersal, y asi veremos en el processo de la obra como triunfa la muerte quando mata a madona Laura que viene en persona de la razon. En el quarto lugar triunfa la fama de la muerte, pues, avn que el cuerpo este apartado de la anima y no pueda obrar, paresce que por la fama de sus obras passadas es tornado de nueuo en la vida, pues viue su nombre por alabança y por exemplo suyo se mueuen otros a virtud, $y$, porque la fama resucita lo que la muerte mata y es mas poderosa que ella, llamamos a este cuarto estado el triumpho de fama. En la quinta parte es mas poderoso el tiempo que la fama, porque, dando muchas bueltas el sol y passando muchos dias meses y años acabanse las memorias donde se conseruauan los hechos famosos, pierdense los libros donde las claras hazañas estauan escritas, caense los edificios en que los nombres magnificos con grandes letreros esculpidos vienen a ser encubiertos de escuras tinieblas y oluido perpetuo. Finalmente toda cosa mortal viene por tiempo a ser corrumpida, y desta manera triumpha tambien de la fama (fol. a3).

Éste es un claro ejemplo de una manipulación en la traducción del comentario. Se aprecia con facilidad que la «doctrina» que Illicino ofrece de un modo seco, intelectual, con la idea de escribir un tratado más bien de carácter filosofico para posiblemente un lector escogido y culto, aquí se transforma en un lenguaje llano, conocido del lector castellano y con un claro afán divulgativo. Vemos ya que hay dos tipos de traducciones de comentarios. La que lleva a cabo el traductor anónimo catalán, que puede ser tomada simplemente como un ejercicio académico, y esta traducción de Obregón que, al recrear las ideas principales con la materia del libro, casi podría decirse que deja a un lado el estilo y la forma de lo que daríamos en llamar una traducción para llegar a algo nuevo, a un texto manejado de una manera libre. Se continúa viendo lo mismo cuando habla del sexto triunfo. Dice lo siguiente: 
El sexto triunpho se llama de la diuinidad, porque despues del juyzio final no correra el sol como agora haze, mas siempre estara parado en vn lugar, de donde se seguira que no aura tiempo passado ni por venir pues siempre sera presente, y desta suerte triumphara la diuinidad del tiempo, segun mas largamente veremos en su lugar; y pues tengo ya dicho a vuestra muy magnifica señoria la materia y sugeto Vniuersal del libro presente, quiero agora dezir le el prouecho que del le podra seguir a quien leyere (fol. a3).

Obregón, aparte de mostrar un interés innegable por su lector, traduce el comentario de una manera distinta. Esto en el contexto peninsular es importante señalarlo porque lo que estoy destacando en este trabajo es algo que no mencioné cuando, al hablar de su traducción en general tomando como base el Triunfo de Amor, ya apunté algunas diferencias entre la traducción del verso y la de la prosa ${ }^{26}$. Obregón, cuando traduce la «doctrina» de Illicino, es mucho menos «fiel» que cuando traduce lo que Illicino dice estrictamente sobre la obra de Petrarca. Según podemos observar, es cierto que pone de manifiesto que los seis triunfos son seis estados del alma, pero también lo es que no habla tanto de un tránsito filosófico. En segundo lugar, nos encontramos con su descripción del Triunfo de Amor:

Muy poderoso finge nuestro poeta que venia amor sobre vn carro trivmphal con infinitos presioneros en muy estrechas presiones.

También, algo parecido lo encontramos cuando Obregón habla del Triunfo del Tiempo, en donde la recreación es evidente, como quedó señalado. Vemos cómo el autor habla de edificios que se caen, de que todo lo humano pasa y las grandes hazañas son olvidadas. No se aleja de un tono de un tono de púlpito, pero es indiscutible que no sigue a Illicino como el comentario catalán. Esta traducción del comentario de Illicino en realidad es un comentario tradicional. El hecho de que recree no significa que rompe esquemas o estructuras ya prefijadas. Es un comentario tradicional. Se puede constatar este asunto cuando se lee lo que la crítica considera las partes de un comentario. En Obregón encontramos todo lo que Quain señala como propio de un comentario:

La costumbre en los comentaristas medievales de autores clásicos de anteponer a sus obras un «esquema» generalmente llamado accessus es conocida desde hace mucho tiempo. En esa nota preliminar se trataban temas como los siguientes: vita auctoris, titulus operis, intentio scribentis, materia operis, utilitas, y qui parti philosophiae supponatur. En diferentes obras el número the estos temas podía abreviarse o expanderse, pero el proposito común de proveer un resumen introductorio a la obra en cuestion está presente en todas las formas de los accessus.

[The custom of medieval commentators on classical authors of prefixing to their works a «schema» generally called an accessus has long been known. In such a prefatory note they treated of items such as the following: vita auctoris,

26 El concepto 'intérprete tan fiel' de Antonio de Obregon*, Bulletin of Hispanic Studies 83, 1996, 225-37. 
titulus operis, intentio scribentis, materia operis, utilitas, and qui parti philosophiae supponatur. In different works the number of these items might be curtailed or expanded, but the common purpose of providing an introductory summary to the work in question, is present in all forms of the accessus. ${ }^{27}$

No se aparta, sino que presenta un texto propio y de más asequible lectura. El contenido filosófico va unido a la utilidad del libro. Se puede ver en el pasaje donde trata precisamente este punto, pues es original pero no se aparta del texto base:

Sentencia muy aprouada es por todos los mortales que las obras virtuosas o la misma virtud sea el sumo bien, y aquella cosa es juzgada por muy prouechosa que por camino derecho nos guia a tal possession; y pues esto asi esta aueriguado, que cosa a nosotros se nos puede mostrar de mas vtilidad del apetito y de la claridad de la razon (fol. a3).

Con respecto al Vellutello, la traducción castellana a su comentario es muy particular. Hernando de Hozes presenta en 1554 una traducción con comentario que ya ha asimilado todas las tendencias aperturistas con respecto a la traducción en la época ${ }^{28}$. Lo que Vellutello presenta como una cuestión de filosofia muy útil al hombre para su propio beneficio espiritual (B iii) ${ }^{29}$, Hozes lo presenta como una recreación en lo que predomina no es la filosofía sino la poética de Petrarca. Deja a un lado las cuestiones del tiempo, las pasiones, y comienza así su comentario:

Todo lo contenido en estos seys Triumphos finge nuestro Poeta auer sido vn suefio o vision, y dize nos agora al principio dellos como le fue mostrada en otro tal tiempo como en el que se auia enamorado de su madona Laura; y que esto fuesse a los seys de Abril el mismo Poeta lo muestra en el Triumpho de la muerte y en alguno de sus sonetos y canciones (fol. 1).

Como puede observarse, en realidad el comentario no es una traducción al gusto latinista. Es totalmente una recreación, es decir, una traducción que se lleva a cabo según el criterio del traductor. Si Obregón se permitía ciertas libertades, Hozes ya transforma el comentario. Se deja de lado una traducción «literal» como la catalana y se llega a la creación de un comentario nuevo, en donde se destacan más las cuestiones poéticas que las de la doctrina. Ése enfasis en lo poético es indiscutible que responde a las modas literarias de la época. Recordemos que estamos en la Castilla que sigue la herencia de Boscán y Garcilaso. Ahora se está presentando al Petrarca poeta ante el lector, lo cual se debe quizá no sólo a la popularidad de Petrarca en la época, sino también a las ideas que sobre la traducción se fueron desarrollando en la Península. Dentro de este amplio panorama hay una figura de capital importancia, que es la de Fernández

27 E.A. Quain, «The Medieval Accessus ad auctores, Traditio 3, 1945, 215-64.

23 Hernando de Hozes, Los trivnphos de Francisco Petrarcha, ahora todo nueuamente traduzidos en lengua castellana, en la medida, y numero de versos, que tienen en el toscano, y con nueua glosa, Medina del Campo: Guillermo de Millis, 1554. Las citas seran de esta edición.

${ }^{29}$ Alexandro Vellutello, Sopra I Triomphi del Petrarca, Venezia: 1545. Las citas son de esta edición. 
de Villegas. Ya en 1516 se atrevió a afirmar abiertamente que para su traducción de los versos habra utilizado información contenida en el comentario que traduce de Landino ${ }^{30}$. Además, llega a decir, haciendo suya la teoría de Alfonso de Madrigal, que es imposible traducir por las mismas palabras:

Débese notar que el Dante escribe su obra en coplas de tercetos (que ansí los nombra el toscano), correspondiente el tercero pié al primero, y despues el primero del terceto siguiente al segundo: de manera que aquellos cuatro farian una media copla de arte mayor, que, como es de ocho piés, viniera ansí justo al talle; pero como escribe de tres en tres, en dos tercetos faltan dos pies para una copla del arte mayor ya dicha: yo probé á los facer-ansí en tercetos, la cual manera no es en nuestro uso; y parescíame una cosa tan desdonada, que lo deje. Quedo el defeto ya dicho de faltar en cada terceto un pié para la media copla, y dos piés en cada una entera; éstos yo acordé de los suplir desta mane$\mathrm{ra}$ : que algunas veces, ocurriendo de mio algund buen pie, que más aclare su texto 6 confirme su sentencia, póngole; y haya paciencia el Dante que en su brocado se ponga algund remiendo de sayal, que más le faga lucir; y el filósofo dice, que de miserrimo ingenio es siempre usar de lo fallado. Otras veces suplo aquellos piés de lo que alguno de sus glosadores dice; y otras también (y las más, cuando buenamente se puede facer) tomo el primero y segundo pic del terceto siguiente, y ansi se facen sus dos, y á las veces tres, tercetos, una copla de ocho piés. Nótese tambien que el que treslada de otro anda tras él con sueltas, y no puede ir cómo ni cuánto quiere; y ansí van, algunas veces, piés algunos forcejados, que no se pudo 6 no se supo mejor facer: rescibase la buena voluntad; que ninguno da más de lo que tiene. Ansimesmo se debe notar que tresladarse una lengua en otra, no solamente en verso, pero ni en oracion soluta, 6 prosa que algunos llaman, es imposible tresladarse por las mismas palabras, que no fuese la más desabrida cosa del mundo; porque en una lengua tiene una cosa gracia, y dicho en otra por aquellas palabras sería muy frio: por ende aqui, en todo cuanto de las mesmas palabras se puede usar, se face; pero en muchas partes se toma el sentido y intencion, más que no las palabras ${ }^{11}$.

Es evidente que Fernández de Villegas en esta cita, siguiendo sin duda a $\mathrm{El}$ Tostado, hace una diferenciación clara entre traducir verso y prosa. Sin embargo, ya ha quedado claro que en su traducción de Dante él ha modificado los versos valiéndose del texto de Landino. Con respecto a su traducción del comentario de este exégeta italiano, hay que dejar en claro que en muchas ocasiones se permite libertades notorias. Un ejemplo lo encontramos al comienzo del Canto V cuando comenta su traducción de los versos de una manera muy diferente a Landino:

30 Cristophoro Landino, Danthe Alighieri fiorentino historiado, Venezia: 1502.

31 Esta transcripción proviene de la edición del siglo XIX que se conserva en la Biblioteca de la Universidad de Cornell: Pero Fernández de Villegas, La Divina Comedia de Dante, Madrid: Tomás Rey, 1868, xi. La signatura actual es PQ/4318/2/136/1868. La transcripción la llevó a cabo el escritor romántico Juan Eugenio Hartzenbusch. Es un ejemplar interesante que debe consultarse. Para el manuscrito, consultese el ejemplar de la Biblioteca Nacional de Madrid cuya signatura es R.30767. Como me ocupo do esta obra y de este ejemplar de la Bibliotoca Nacional de Madrid en otro trabajo, aqui me ha parecido válida la transcripción de Hartzenbusch, que no deja de ser una curiosidad bibliográfica. 
Mas donde mas llanto y dolor se sentra: en el texto del Dante dize y mas pungian guayo; ya diximos como se dize en lengua ytaliana y lo vsa este auctor dezir este vocablo guay y guayo, mas que en nuestra lengua es vicablo muy util y no hemos de vsar del; de la gravedad y dolor de la pena de estos dañados carnales este auctor no pone sino fuego y ayre, y tempestad ardiente que los lleuaba de vnas partes a otras para demostrar la ligereza y inconstancia; mas en la verdad no solamente fuego ay en aquellas infernales penas, segund en muchos logares de la Sagrada Escriptura se dize del fuego, mas ay otras muchas penas y tormentos intolerables; asy mentales y del espiritu, como tan bien corporeas y sensibles para las animas, y despues de su triste resurrecion tan bien para los cuerpos; de las espirituales es la carencia perpetua de la vision de Dios y de la gloria eternal que les es mas penosa que no el fuego (fol. $\mathrm{i}$ ii).

Se trata de toda una amplificación sobre el fuego, que no aparece ni en Dante ni en Landino. Fernández de Villegas hace una manipulación del texto italiano que llega a crear escuela en el ámbito castellano, pues es el mismo método que más tarde seguiría Hernando de Hozes. En Fernández de Villegas es algo típico y en Castilla es algo nuevo. El pasaje con los versos de Dante es comentado de una manera muy distinta por Landino, según se ve en el folio 31 de su comentario $^{32}$. En lo que a traducción se refiere Fernández de Villegas está en la línea de los traductores europeos. Dado que su traducción encierra una interpretación por su parte, se le puede relacionar con ellos, dado que éstos efectuaban el mismo proceso según indica Rita Copeland ${ }^{33}$. Con respecto a lo que aquí estamos tratando, la misma idea aparece también en Carne-Ross, pero de una manera mucho más clara, al afirmar que la traducción verdadera es más que nada un comentario y no un sustituto de ese texto, y además su papel es interpretativo ${ }^{34}$.

Puede afirmarse que los comentaristas italianos sufren un transvase que presenta varias características. Haciendo un resumen, se deben citar tres: 1) supresión de personajes históricos y mitológicos; 2) mentalidad de divulgación popular frente a erudición filosofica para unos elegidos; 3 ) recreaciones individuales y de diversas índoles con fines muy concretos (Villegas). Estas traducciones ponen en primer plano, entre otras cosas, el valor que los traductores le daban a su público y, por lo que a Castilla se refiere, es algo hasta ahora no dicho y muy a tener en cuenta.

Por los motivos arriba expuestos, las traducciones de comentarios también deben considerarse fundamentales para comprender el método de traducción que se siguib durante un período de tiempo. Está claro que hay una diferencia entre traducir verso y prosa. Debe señalarse el significado que tenía la práctica de la traducción en cada época y en cada lengua. Es necesario salirse de los encasilla-

32 Landino, Danthe...

${ }^{33}$ Rita Copeland, Rhetoric, Hermeneutics and Translation in the Middle Ages: Academic Traditions and Vernacular Texts, Cambridge Studies in Medieval Literature 11, Cambridge: Cambridge University Press, 1991, 10-27.

34 D.S. Carne-Ross, *Translation and transposition*, The Craft and Context of Translation, eds. William Arrowsmith y Roger Shattuck, Austin: U of Texas P, 1961, 3-21. 
mientos de «literal» y «libre», dado que el asunto es más complicado y su base son conceptos bastante concretos. Un ejemplo de esto es el papel que el traductor le da a su público. Desde esta perspectiva hay que replantearse el estudio de textos que hasta ahora han sido arrinconados o catalogados descuidadamente. Quizá lo que verdaderamente debe estudiarse son estas producciones que constituyen un ejemplo de una manera peculiar y determinada de traducir y de toda una problemática en la Península.

\section{BIBLIOGRAFIA}

CARNE-Ross, D.S., *Translation and transposition», The Craft and Context of Translation, eds. William Arrowsmith y Roger Shattuck, Austin: U of Texas P, 1961, 3-21.

Cartagena, Alonso de, la Rethorica de Marco Tulluo Cicerón, ed. Rosalba Mascagna, Napoli: Liguori, 1969.

COPELAND, RrTA, Rhetoric, Hermeneutics and Translation in the Middle Ages: Academic Traditions and Vernacular Texts, Cambridge Studies in Medieval Literature 11, Cambridge: Cambridge University Press, 1991.

Fernandez de Villegas, Pero, La Divina Comedia de Dante, Madrid: Tomás Rey, 1868.

HOZES, HERNANDO DE, Los trivnphos de Francisco Petrarcha, ahora todo nueuamente traduzidos en lengua castellana, en la medida, y numero de versos, que tienen en el toscano, y con nueua glosa, Medina del Campo: Guillermo de Millis, 1554.

ILLICINo, BERnaRdo, Triomphi de meser Francesco Petrarca, Venezia: 1475.

LANDINO, CRISTOPHORO, Danthe Alighieri fiorentino historiado, Venezia: 1502.

MENA, JUAN DE, Obras completas, ed. Miguel Angel Pérez Priego, Barcelona: Planeta, 1989.

MorReAle, MARGherTta, Castiglione y Boscán: el ideal cortesano en el renacimiento espaniol, 2 vols., Madrid: BRAE, 1959.

OBREGON, ANTONIO DE, Francisco Petrarca, con los seys triunfos del toscano sacados en castellano con el comento que sobrellos se hizo, Logroño: Arnau Guillén de Brocar, 1512.

Palencia, Alfonso De, Batalla campal de los pernos, Sevilla: 1490

Petrarca, Francesco, Triumphi, ms. 534 de la Biblioteca Nacional de Paris.

QuaIN, E.A., «The Medieval Accessus ad auctores», Traditio 3, 1945, 215-64.

Rico, Francisco, Alfonso el Sabio y la General Estoria, Barcelona: Ariel, 1984.

Recio, RoXaNA, Alfonso de Madrigal (El Tostado): la traducción como teorfa entre lo medieval y lo renacentista», La Corónica 19.2, 1992, 112-131.

, «as interpolaciones latinas de Sor Isabel de Villena: ¿traducciones, glosas 0 amplificaciones?», Anuario Medieval 5, 1993, 126-40. 
- «La interrelación intelectual en la Península: Santillana y Ferrer de Blanes», Anuario Medieval 6, 1994, 159-73.

, $* \mathrm{El}$ concepto de la belleza de Alfonso de Madrigal (El Tostado): la problemática de la traducción literal y libre», La traducción en Espanta (ss. XIV-XVI), ed. Roxana Recio, León: Universidad de León, 1995, 59-68.

, «El concepto 'intérprete tan fiel' de Antonio de Obregon», Bulletin of Hispanic Studies 83, 1996, 225-37.

, «a literalidad y el caso de la Cárcel de Amor: el quehacer del traductor catalán y del traductor italiano», Hispanic Journal 17.2, Fall 1996, 271-83.

, «'Por la orden que mejor suena': traducción y Enrique de Villena», La Coronica 24.2, Spring 1996, 140-53.

*Del latín al vernáculo: la difusión peninsular del Decamerón », Livius 9, 1997, 109-19.

, «Humanismo y exégesis medieval: el caso de Ferrer de Blanes $\$$, Actas del XII Congreso de la Asociación Internacional de Hispanistas; Birmingham, 1995, ed. Aengus M. Ward, 5 vols., Birmingham: The University of Birmingham, 1998, vol. 1, 293-301.

, «Approaches to Medieval Translation in the Iberian Peninsula: Glosses and Amplifications, Fifteenth-Century Studies 1998, en prensa.

, «Puntualizaciones sobre la traducción catalana del Triunfo de Amor de Petrarca según el manuscrito 534 de la Biblioteca Nacional de Paris», Actas del XIII Congreso de la Asociación Internacional de Hispanistas; Madrid 1998, en prensa.

Russell, PETER, Traducciones y traductores en la Península Iberica (1400-1550), Bellaterra: Universitat Autónoma de Barcelona, 1985.

SAINT JÉROME, «A Pammachius; la meilleure méthode de traduction», Lettres, ed. Jérome Labourt, 8 vols., Paris: Les Belles Lettres, 1953.

Santoyo, Juluo Cesar, Teoria y critica de la traduccion: Antologia, Bellaterra: Universitat Autonoma de Barcelona, 1987.

Vellutello, Alexandro, Sopra I Triomphi del Petrarca, Venezia: 1545.

WeIss, Julian, The Poet's Art: Literary Theory in Castile c. 1400-1600, Medium Aevum New Series 14, Oxford: Oxford University Press, 1990. 